University of South Florida

DIGITAL COMMONS

Digital Commons @ University of

@ UNIVERSITY OF SOUTH FLORIDA

South Florida

Psychology Faculty Publications

Psychology

$3-2013$

\title{
Functional Segregation of the Entopallium in Pigeons
}

Robert G. Cook

Tufts University

Tadd B. Patton

Augusta State University

Toru Shimizu

University of South Florida, shimizu@usf.edu

Follow this and additional works at: https://digitalcommons.usf.edu/psy_facpub

Part of the Psychology Commons

\section{Scholar Commons Citation}

Cook, Robert G.; Patton, Tadd B.; and Shimizu, Toru, "Functional Segregation of the Entopallium in Pigeons" (2013). Psychology Faculty Publications. 414.

https://digitalcommons.usf.edu/psy_facpub/414

This Article is brought to you for free and open access by the Psychology at Digital Commons @ University of South Florida. It has been accepted for inclusion in Psychology Faculty Publications by an authorized administrator of Digital Commons @ University of South Florida. For more information, please contact digitalcommons@usf.edu. 
Published in final edited form as:

Philosophy. 2013 March ; 130: 59-86.

\title{
Functional Segregation of the Entopallium in Pigeons
}

\author{
Robert G. Cook ${ }^{1}$, Tadd B. Patton ${ }^{2}$, and Toru Shimizu ${ }^{3,{ }^{*}}$ \\ ${ }^{1}$ Department of Psychology, Tufts University \\ 2Department of Psychology, Augusta State University \\ ${ }^{3}$ Department of Psychology, University of South Florida
}

\section{Abstract}

In birds, the entopallium is the primary telencephalic target of the major visual ascending route called the tectofugal pathway. Often functionally compared to the primate geniculo-striate pathway and its subsequent telencephalic (cortical) regions, the latter processes visual information in a parallel fashion in terms of anatomy, physiology, and function. Little is known, however, about the exact mechanism of whether or how information is segregated or integrated in the avian tectofugal pathway including the telencephalon. Testing four pigeons, we examined whether or not color, form, and motion information is selectively processed by different portions of the entopallium. Each learned three distinct visual tasks requiring discrimination of different combinations of color, form and motion cues. After learning and pre-lesion testing, two pigeons received lesions to the anterior portion of the entopallium and two received lesions to the posterior entopallium. During post-lesion testing the pigeons with anterior lesions exhibited significant deficits in those tasks most dependent on color and form discrimination, but showed no deficit on a task that had involved discriminating among forms that were moving. Pigeons with posterior lesions showed a different pattern of deficits, exhibiting significant reductions in discriminating both moving and static forms, but little or no deficits in color discrimination. These divergent profiles of effects for each lesion suggest there is a functional segregation of visual information processing in the pigeon telencephalon. This indicates a convergence between birds and primates regarding the parallel processing and separation of information within their phylogenetically distinct major visual pathways.

\section{Keywords}

birds; visual system; lesion

\section{Introduction}

Birds, along with mammals, are the major classes of highly visual vertebrates on the planet (Lazareva, Shimizu, \& Wasserman, 2012). Despite the unquestioned importance of the visual modality to birds, the severe weight restrictions of muscle-powered flight has markedly limited the overall size of birds and their visual nervous system. The entopallium

\footnotetext{
"Corresponding author: Toru Shimizu, Department of Psychology, PCD 4118G, University of South Florida, 4202 E. Fowler Avenue, Tampa, FL 33620-7200, Phone \#: 813-974-0352, FAX \#: 813-974-4617, shimizu @usf.edu.
} 
is one of the main visual centers in the avian forebrain. It is the telencephalic target of the tectofugal (or collothalamic) visual route, which travels from the retina to optic tectum to thalamus then to the entopallium (Karten \& Hodos, 1970). Pioneering work by Watanabe and others demonstrated that lesions in the entopallium, as well as relaying centers in the tectofugal pathway, result in severely diminished perceptual effects in pigeons, including discrimination of color, pattern, and brightness (Hodos, 1969, 1993; Hodos \& Karten, 1966; Hodos, Weiss, \& Bessette, 1988; Watanabe \& Bischof, 1997; Shimizu \& Watanabe, 2012).

Because the tectofugal pathway plays an essential role in visual behavior of birds, it is functionally compared to the predominant retino-geniculo-striate pathway in primates although phylogenetically, they are distinct (Shimizu \& Bowers, 1999). In the primate geniculo-striate system and subsequent cortical regions, visual information is processed in separate streams in a parallel fashion in terms of anatomy, physiology, and function (Ungerleider \& Mishkin, 1982; Baizer, Ungerleider, \& Desimone, 1991; Goodale \& Milner, 1992; Milner \& Goodale, 2006). There have now been a number of proposals for the nature of these divisions. For instance, Ungerleider and Mishkin (1982) proposed that "where" information (e.g., motion perception) is primarily processed in the dorsal stream in the cerebral cortex, which receives input mostly from magnocellular neurons located in the lateral geniculate nucleus. In contrast, "what" information (e.g., color and form perception) is predominantly processed in the ventral stream, which depends on neuronal input from the parvocellular division (Ungerleider \& Mishkin, 1982). Are avian visual system similarly divided into functional streams? If so, do these share any common mappings with those proposed for the mammalian visual system?

Several anatomical and physiological studies show that the thalamic center of the avian tectofugal pathway, the nucleus rotundus, processes visual information in a parallel fashion. For instance, the anterior and posterior subdivisions of the nucleus rotundus respectively receive input from different layers of the optic tectum, suggesting that there are anatomical parallel channels between the tectum and nucleus rotundus (Mpodozis et al., 1996; Karten, Cox, \& Mpodozis, 1997). Moreover, physiological recording studies show that neurons of these rotundal subdivisions have different response characteristics. Specifically, colorsensitive neurons and luminance-sensitive neurons are mainly observed in the anterior portion of the nucleus rotundus, whereas many motion-sensitive neurons are found in the posterior portion (Wang, Jiang, \& Frost, 1993).

Little is known about whether these parallel channels extend from the nucleus rotundus to the entopallium in the telencephalon. A few connection studies, however, show that a general connection pattern between the thalamus and telencephalon is organized topographically along the anterior-posterior axis. Neurons in the anterior rotundus tend to project to the anterior portion of the entopallium while the posterior rotundus primarily projects to the posterior entopallium (Laverghetta \& Shimizu, 2003; Krützfeldt \& Wild, 2004, 2005).

Based on these anatomical results, Nguyen et al. (2004) examined the anterior-posterior differences of the entopallium by looking at the behavioral effects of selective lesions. Using pattern detection task in which spatial gratings had to be detected in visual noise and a 
coherent motion task in which dot stimuli either moved in a coherent direction or not, Nguyen et al. (2004) found that lesions in the anterior portion selectively impaired performance in the pattern detection task whereas, lesions in the posterior portion impaired performance in the coherent motion task. Based on these results, these authors suggested that the anterior and posterior entopallium are involved in processing spatial pattern and motion perception, respectively. Their study is the first and only empirical work showing that a parallel visual processing mechanism extends from the thalamus to the telencephalon in non-mammals. If this finding is confirmed, it has significant meaning for our understanding of the evolution of the vertebrate visual system by suggesting that phylogenetically distant animals independently evolved to have a similar parallel mechanism to process visual information. Because of its important implications, the present study further investigated this potential functional segregation of the entopallium.

The idea underlying the present approach was to evaluate the effects of the different lesions by testing the pigeons with a broad palette of tasks using different types of visual stimuli. By testing more types of stimuli we can develop more complete visual profiles of the deficits induced by each type of lesion to different regions of the entopallium. Our concern was that the traditional approach of using a single task for this evaluation possibly misses effects or causes them to be tied specifically to the nature of the stimuli or responses require of a specific task. With this ambitious objective for the present experiment, we trained four pigeons to concurrently perform three different visual tasks concurrently. All stimuli were presented to the birds on a computer monitor in a touchscreen-equipped testing chamber. As described next, each task involved different response contingences and stimuli. Taken together, these tasks and associated visual stimuli capture a more complete and detailed picture of any functional segregation.

One task was a texture target localization task. In this task, the pigeons were trained to visually search for an odd "target" region of elements that was randomly located within a larger matrix of dissimilar "distractor" elements (Cook, 1992a, 1992b; Cook, Cavoto, Katz, \& Cavoto, 1997; Cook, Cavoto, \& Cavoto, 1996). The pigeon's task was to locate and peck at the odd target region to obtain food (see example texture in Figure 1). It had been established that the pigeons' processing of these hierarchically-organized displays bears a resemblance to those observed in humans, suggesting that similar underlying mechanisms are involved (Cook, 2000; Cook \& Hagmann, 2012). In the present study, the pigeons had to locate targets that were defined by either color or shape differences from the surrounding regions (see Figure 1). This task evaluates how the lesions likely affected the global visual grouping of these two separate dimensions.

The second task was a dynamic shape discrimination task. In this task the pigeons had to discriminate the internal angle $\left(95^{\circ}\right.$ vs. $135^{\circ}$, see examples in middle of Figure 1) of two different double triangle outline figures in a go/no-go procedure (cf. Kellman \& Short, 1987). These two forms or shapes were presented at random orientations at different locations on the monitor, thus requiring the birds to process the global features of the shapes. If the $\mathrm{S}+$ shape was presented the pigeons were reinforced for pecking at it, while the $\mathrm{S}$ shape was tested in extinction. To evaluate the contribution of motion to this shape discrimination, these stimuli were presented in three ways. One involved coherently rotating 
the stimuli around its principal axis. The second involved presenting the same views, but in a randomized sequential order. The third involved presenting the stimuli as static nonmoving forms. We have consistently found that coherent presentation facilitates the discrimination of form and motion (Asen \& Cook, 2012; Cook \& Roberts, 2007; Cook, Shaw, \& Blaisdell, 2001; Koban \& Cook, 2009). From unpublished studies, we have found that the coherent presentation of these shape stimuli generally results in a slightly better discrimination in comparison to the random or static methods of presentation. Consequently, this task allowed us to evaluate how the different lesions affected the discrimination of shape as a function of different movement presentation methods and training.

The third task was a form of a "where" and "what" shape discrimination task. The inclusion of this task allowed us to evaluate how the lesions affected the discrimination of multiple shapes as influenced their relative identity and location. It was derived from the empirical work of Kirkpatrick-Steger and Wasserman (1996) on pigeons. It involved a 2-alternative choice task that required attention to the what/where properties of the multiple objects present within a display. In this task to obtain food, the pigeons had to choose which of two displays had the correct combination of objects and in their proper locations. The lower portion of Figure 1 shows one set of the stimuli tested. In the task, one stimulus was designated as correct for each bird (e.g., top left). If paired with stimuli as a foil from the top row, it requires the pigeon to discriminate the identity or the "what" properties of the objects present. If paired with stimuli along the left column, the pigeon must discriminate the location or the "where" properties of the objects since the objects are otherwise the same. When paired with any of the remaining nine stimuli, of course, they differ redundantly with regards to both their what and where properties.

The pigeons were first sequentially trained to perform each task. Following this separate training, the tasks were then combined and tested concurrently with one task tested each day in rotation. The last 24 sessions of this concurrent testing period (eight per task) was used to measure pre-lesion discrimination performance. Following surgery and recovery, a comparable period of 24 sessions measured post-lesion performance. The comparison of the pre- and post- lesion performance allowed us to create a profile of how the different lesions affected the visual profile of the pigeons and help isolate any potential functional segregation of the entopallium.

\section{Method}

\section{Subjects}

Four male adult White Carneaux pigeons (Columba livia) were tested. The birds were maintained at $80-85 \%$ of their free-feeding weight during testing with free access to water and grit in their home cages, which were housed in a colony room with a 12-h light-dark cycle.

\section{Apparatus}

Testing was conducted in a flat-black Plexiglas chamber. Stimuli were presented by computer on a CRT color monitor visible through a $26 \mathrm{~cm} \times 18 \mathrm{~cm}(\mathrm{~W} \times \mathrm{H})$ viewing 
window in the middle of the front panel of the chamber. Pecks to the monitor were detected by an infrared touch screen. Mixed grain was delivered through a centrally placed food hopper under the monitor and a 28 -volt houselight was centrally located in the chamber ceiling.

\section{Behavioral procedures}

Textured target localization task-In this task, the pigeons were trained perform a standard target localization discrimination with textured stimuli (e.g., Cook, 1992a, 1992c). The details of this procedure are widely available and not as extensively described as for the other tasks. The pigeons' task was to locate and peck the contrasting target region, defined by either color or shape regional differences, from a surrounding region of distractors to obtain food reinforcement. Texture displays consisted of 384 small colored shape elements arranged in a $24 \times 16$ rectangular matrix. Elements of the texture displays were created from randomized pairwise combinations of 8 colors and 8 shapes (3-6 mm in size). The target region consisted of a $7 \times 7$ block of elements randomly located on each trial within the larger matrix of surrounding distractor elements and differed either in color or shape (see examples in Figure 1). Chance performance was $35 \%$ for these birds as determined empirically from uniform "targetless" control displays.

Trials began with a peck to a circular white ready signal that was replaced by texture display. If a pigeon first accumulated five pecks within the target region (including the immediately surrounding row and column of distractor elements) an accurate localization response was judged to have occurred and reinforced ( $2.5 \mathrm{~s}$ of grain access). Instead, if a pigeon first accumulated five pecks to the distractor region, the response was consider incorrect, the display was turned off, and the overhead houselight extinguished for $10 \mathrm{~s}$. Trials were separated by an illuminated 1-s inter-trial interval. Each session consisted of 140 trials ( 70 color / 70 shape trials)

Dynamic shape discrimination task-In this task, the pigeons were tested in a go/nogo discrimination requiring them to distinguish between two opposing base-aligned outlined triangles whose interior angle at their intersection varied $\left(95^{\circ}\right.$ vs. $135^{\circ}$; see examples in Figure 1). The location of the stimulus was randomized on the monitor within a central $11 \times$ $11 \mathrm{~cm}$ area of the viewing window on each trial. Stimuli were presented at three different sizes (height $\times$ base each one triangle; $3.5 \times 2.8,1.5 \times 1.2,1 \times .8 \mathrm{~cm}$ ). The lines for all sizes were approximately $1 \mathrm{~mm}$ in width and colored red and presented on a black ground. The orientation of each stimulus was randomized along both the $\mathrm{x}$ and $\mathrm{z}$ axes $\left(360^{\circ}\right.$ in $1^{\circ}$ divisions).

Finally, three different modes of presenting each trial's stimulus were tested. In the dynamic condition, the stimulus smoothly rotated around the picture plane's y axis at a rate of .83 rotations per second in $30^{\circ}$ increments for the entire duration of the trial. In the random condition, successive views changed every $0.1 \mathrm{sec}$ were randomly drawn with replacement from the 360 different orientations of the stimulus around this same axis for the entire duration of the trial. In the static condition, the stimulus was presented at a single randomly determined orientation from the same y axis rotation for the entire duration of the trial. The 
starting orientation of the dynamic and random conditions was randomly determined on each trial.

Trials began with a peck to a circular white ready signal, and then replaced by a single discriminative stimulus $\left(95^{\circ}\right.$ or $\left.135^{\circ}\right)$ for $20 \mathrm{~s}$. Half the pigeons were reinforced for pecking at the $95^{\circ}$ stimuli and the other half were the $135^{\circ}$ stimuli. Pecks during S+ trials were reinforced (2.8 s of grain access) on a variable-interval 10-s schedule. Pecks to S- stimuli were punished with a response-dependent timeout, in which the overhead houselight was extinguished for 1-s for each peck during the presentation interval. After either consequence, a 3-s inter-trial-interval occurred. Each session consisted of 96 trials. Each of the three presentation conditions was tested 32 times ( $18 \mathrm{~S}+/ 18 \mathrm{~S}-)$ at each size. A small percentage of non-reinforced probe trials were conducted to allow for the uncontaminated measurement of peck rate without the presence of food presentations. Each presentation condition at each size was tested once as probe trial each session.

Simultaneous "where/what" discrimination task-In the third task, the pigeons were tested in a two-alternative simultaneous discrimination that required them to discriminate between stimuli that varied in the "what" and "where" features of the shapes comprising the pictures. The stimuli consisted of three sets of 16 drawings that each contained two shapes with them (see bottom row Figure 1). Each stimulus contained two object-like 3D shapes (wedge, cylinder, cone $\&$ handle) positioned in different spatial relations (right, left, above, and below) in an $8 \mathrm{~cm} \times 6.4 \mathrm{~cm}$ white background. Each shape occupied an area of between 2 to $3 \mathrm{~cm}^{2}$. One common shape was paired with each of the other three shapes in four possible relative positions. A different combination of shape and position was designated as correct for each bird, with the remaining 15 other stimuli serving as incorrect foils for that subject. By comparing performance against the three foils that contained the same objects as the correct stimulus, but in different positions, it was possible to obtain information about how the spatial position of the objects was used (the "where" condition). By comparing performance against the three foils that contained a different object from the correct stimulus but in the same positions, it was possible to obtain information about how the identity of the object was used (the "what" condition). The remaining nine foils differed in both their where and what properties (the "redundant" condition) relative to the correct stimulus.

Finally, these stimuli were tested using three different looking stimulus sets. The first set was a direct replication of Kirkpatrick-Steger and Wasserman's (1996) stimuli using outlines of the four shapes directly adjacent to each other. The second set was similar, but the objects were separated by approximately $1 \mathrm{~cm}$ at their front edges. These removed the occlusion present between the objects in the first set. The third set using the same shapes, but these were rendered three-dimensionally with shaded and filled blue surfaces rather than with outline contours (Bryce 4 modeling software).

Trials began with a peck to a circular white ready signal that was replaced by two stimuli (one correct/ one foil). These were presented at random, non-overlapping positions within an $800 \times 400$ pixel central viewing area. The pigeon indicated its choice among the two stimuli by pecking at one eight times. The choice to the correct stimulus resulted in food reward 
( $2.8 \mathrm{~s}$ of grain access), while the choice of the incorrect stimulus was punished with a 5-s dark timeout. After either consequence, a 3-s inter-trial-interval occurred. Each session consisted of 135 trials. Each of the three stimulus sets were tested 45 times with the correct stimulus paired with each of the 15 incorrect stimuli once within each stimulus set. The order of these trials was randomized each session.

Training-The pigeons were sequentially taught each of these tasks during the ten months prior to surgery. They first learned the "where/what" discrimination task (approximately 6080 sessions of choice training), followed by the dynamic shape discrimination task (50-70 sessions of training), and finally the textured target localization task (approximately 25-40 sessions of training). After learning each task, analytic tests of varying types and duration for each task were conducted, but are not included here. After all three discriminations had been successively acquired; they were all reintroduced and tested concurrently in threesession blocks (one task per session). Prior to surgery, each pigeon had been tested for at least eight 3-session blocks of concurrent training. During the latter stages of this pre-lesion baseline phase, one bird was mistakenly assigned a new correct stimulus for its where/what discrimination. It was in the process of relearning this variation of the task at the time when the lesions had to occur. As a result, this bird's accuracy on the where/what task was lower than it had previously been during original learning.

\section{Post-lesion procedures}

Lesions-Once birds reached the criterion, bilateral electrolytic lesions were administered to either the anterior or posterior portion of the entopallium ( 2 animals each). For the lesion surgery, pigeons were deeply anesthetized with ketamine $(40 \mathrm{mg} / \mathrm{kg}$ of body weight, i.m. Aveco Co., Inc., Fort Dodge, IA) and xylazine (10 mg/kg of body weight, i.m. Lloyd Laboratories., Shenandoah, IA), and were then placed in a stereotaxic apparatus (David Kopf Instruments, Tujunga, CA). All lesions were administered through an insulated electrode attached to a D.C. Constant Current Lesion Maker (Grass model \# D.C. LM5A, Quincy, MA) at a current of $1 \mathrm{~mA}$ for 20 seconds. The location of lesion target was specified according to coordinates in the stereotaxic atlas of the pigeon brain (Karten \& Hodos, 1967). Lesion electrodes were made by coating insect pins with epoxy (EPOXYLITE Corp., Irvine, $\mathrm{CA})$ and then exposing the tip $(0.5 \mathrm{~mm})$. After surgery, the pigeon was returned to its cage for recovery and monitored daily for seven days.

Behavioral Testing-After the recovery period, the birds were tested again in the same three tasks. Each bird was tested for 24 sessions in eight 3 -session blocks (one task per session).

Tissue Processing-After the completion of the post-operative tests, the pigeons were deeply anesthetized via ketamine (at least $40 \mathrm{mg} / \mathrm{kg}$ of body weight, i.m.) and xylazine (at least $10 \mathrm{mg} / \mathrm{kg}$ of body weight, i.m.) and then perfused transcardially with a $0.9 \%$ saline solution followed by $4 \%$ ice-cold paraformaldehyde in phosphate buffer (0.1 M PB, pH 7.4). Brains were post fixed for 12 hours at $4^{\circ} \mathrm{C}$, followed by immersion in a $30 \%$ sucrose solution for 24 hours at $4^{\circ} \mathrm{C}$. Brains were then frozen in dry ice and cut in $40 \mu \mathrm{m}$ transverse sections on a sliding microtome. Sections were washed three times at 10 min. each in PB 
then mounted on slides and Nissl stained with cresyl violet. Finally, tissue mounted slides were dehydrated with ethanol, cleared with xylene, and coverslipped.

Lesion reconstruction-Entopallial reconstruction was carried out on a series of standard plates derived from the atlas of the pigeon brain of Karten and Hodos (1967). Gliosis and necrosis in the telencephalon was recorded. The extent of each lesion was determined from measurements of reconstructions made with camera and graphics software (CANVAS. v.11.0).

\section{Results \\ Lesions}

Microscopic examinations revealed that all four birds had lesions primarily confined in the entopallium, but in some cases, slight damage was seen in neighboring regions, including the nidopallium, mesopllium, and lateral striatum. Two birds (\#3H \& \#4H) were categorized in the anterior lesion group since the majority of destroyed area was observed anterior to A10.0 based on the pigeon brain atlas (Karten \& Hodos, 1967). The other two (\#1A \& 2H) belonged to the posterior lesion group based on more posterior damage. Figure 2 shows examples of each type of lesion.

\section{Textured target localization task}

The results of the pre-lesion and post-lesion testing in the textured target localization task are shown for each lesion group in Figure 3. Performance was measured and compared using each bird's accuracy at locating color and shape targets during the eight pre-lesion and post-lesion target localization sessions.

The birds receiving an anterior lesion showed a substantial disruption of their localization of both color and shape targets (see top panel of Figure 3). T-tests comparing localization accuracy between pre-lesion and eight post-lesion sessions confirmed that both anterior birds individually showed significant declines in their localization of color targets, both $t$ s (14) $>3.5$ (all statistical tests in this paper were evaluated using an alpha level of $P<.05$ ). Both birds also showed numerical declines in their localization of shape targets after the surgery as well. However, this was significant only for bird, $t(14)=4.34$ and not the other, $t(14)=1.53$. Part of the reason for latter was due to the fact that this bird's shape performance recovered quickly, unlike its color performance. During the first two post-lesion sessions, for example, this bird's shape performance declined considerably ( $64 \%$ to $43 \%$ ), but recovered to near baseline shortly after that.

Both anterior birds also exhibited changes in how quickly their made their initial response to the display. Both birds responded more quickly to the displays following the lesions. This was true for searches for both color and shape targets. This effect can be seen in the upper left panel of Figure 4. This panel shows the cumulative reaction time (RT) distribution for all trials combined from both pigeons. The leftward shift of the distribution shows the speeding up of responding caused by the lesion. Comparisons of the mean RT from these trials (excluding long RTs $>3500 \mathrm{~ms},<2 \%$ of the data) confirmed that both individual pigeons were significantly faster post-lesion than pre-lesion in responding to the display, 
both $t \mathrm{~s}(14)=3.02$. Although RTs for both correct and incorrect response were faster postlesion, this speeding up of responding was clearly more marked for incorrect responses (988 vs. $1208 \mathrm{~ms}$ ) than correct ones (938 vs. $1019 \mathrm{~ms}$ ).

The birds with posterior lesions birds exhibited a different pattern of effects from the anterior group. After their lesion, there was a clear disruption in their ability to localize shape targets, but there almost no impact on the localization of color targets. For shape targets, both birds showed significant decreases in accuracy over the eight post-lesion sessions compared to those from before the lesion, both $t s$ (14)>3.41. Overall, their level of post-lesion shape accuracy was comparable to what was observed with the anterior birds.

In contrast to the anterior birds, their capacity to find the color targets was not greatly affected by the lesion. One bird showed no drop in accuracy in localizing color targets $t(14)=0.34$, being just as accurate after as before the lesion. The other bird showed a significant post-lesion decline in color performance, $t(14)=2.18$, although this was in part because this bird was so good at the color task before the lesion. It remained quite good after the lesion, as well. When we compared just post-lesion color and shape performance between the two groups of birds using mixed design ANOVAs (Group $\times$ Session), we found that post-lesion color accuracy was significantly higher in the posterior group than the anterior group, $F(1,2)=293.3$. This was not the case for shape targets, where post-lesion shape accuracy was equivalent across the two groups.

Finally, unlike the anterior group, both posterior birds showed no large change in how quickly they responded to the displays, if anything, they slowed just a bit. This can be seen in the upper right panel of Figure 4. Again this panel shows the cumulative RT distribution for all pre and post-lesion trials combined from both pigeons. Comparisons of the mean prelesion and post-lesion RTs from these trials (excluding long RTs > $3500 \mathrm{~ms}$ ) found these were equivalent for one bird. The second bird showed a significant slowing in RT postlesion, 892 to $1011 \mathrm{~ms}, t(14)=4.2$.

\section{Dynamic Shape task}

The results with the dynamic shape discrimination task contrasted with those of the texture localization task. Whereas in the latter task, the two anterior pigeons showed marked disruption of both color and shape accuracy and overall faster responding, in this task they showed little disruption in their discrimination. The results of the pre and post-lesion testing with the dynamic shape discrimination are separately shown for the two lesion groups in Figure 5. The bars show the average discrimination ratio for each condition, while the individual symbols again show the results for the individual birds. Performance was measured using each bird's discrimination ratio in each session using the number of pecks emitted during the last 5 seconds of each presentation. Discrimination ratios for each session was calculated by taken the mean $\mathrm{S}+$ peck rate over the last five seconds of each presentation for that session and dividing it by the average number of pecks emitted on both S+ and S- trials combined.

Unlike with the localization task, the birds receiving an anterior lesion maintained their discrimination after the surgery (see top panel of Figure 5) with little or no drop in 
performance. Across all three types of presentation methods (dynamic, random, \& static), the two anterior birds showed equivalent levels of shape discrimination when comparing the eight pre-lesion sessions with the eight post-lesion sessions, all six $t \mathrm{~s}(14)<1.86$ on tests conducted individually with each bird for each condition.

In contrast, the discrimination of both birds receiving posterior lesions showed large drops in their discrimination of these shape stimuli (see bottom panel of Figure 5). This was true across all three types of presentation. Comparing the eight pre- and post-lesion sessions for each presentation condition separately, both birds showed significant reductions in discrimination, all six $t \mathrm{~s}(14)>2.3$.

\section{Simultaneous "where/what" task}

Unlike the first two tasks which showed differential impacts of the respective lesions in each task, choice accuracy in the where/what task was disrupted about equally by either lesion. The results of the pre and post-lesion testing with the where/what discrimination are shown separately for the two lesion groups in Figure 6. Each panel shows choice accuracy for the where, what, and redundant conditions separately. There were no large differences between the three stimulus sets tested (occluded, separated, rendered) and the results are collapsed across this variable.

Collapsed across the where, what and redundant, conditions, all four birds showed significant declines in overall accuracy comparing the eight pre and post-lesion sessions, $t \mathrm{~s}(14)>2.8$. When divided out by these conditions, all four birds showed significant reductions for where and what information that was redundantly presented together, all $t \mathrm{~s}(14)>2.16)$. Further, both anterior birds also showed significant reductions in accuracy when just the where and what conditions was examined separately, ts(14)>2.24).

The results for the posterior birds were more complicated. One bird showed a significant reduction, $t(14)=.3 .3$, in the redundant condition, but no significant effects for the where and what conditions in isolation. The latter result was due to the fact that this bird showed large deficits for these conditions, but only over the first two sessions of post-lesion testing. Why this bird the redundant performance was consistently suppressed relative to where and what accuracy is not clear. The other posterior bird's results are complicated for this specific task by the fact that it was still improving its discrimination at the time of surgery and testing due to accidently change in its assigned correct choice. Its post-lesion accuracy in the where and what conditions was numerically lower than before the lesion, but session-to-session variability reduced the power of the test, and neither was statistically reliable. Consequently it difficult is to reach a firm statistical conclusion about the effects of the posterior lesion from the performance of these two birds. Nonetheless, it is pretty clear that that the lesion impacted and reduced their accuracy with this shape discrimination especially as measured across all conditions. Any judgment, however, regarding the specificity of these effects on the isolated where and what conditions is best reserved for future studies.

Finally, examinations of RT for the two groups of birds in this task revealed no shift in the distribution of pre and post-lesion RTs. This can be seen in the lower panels of Figure 4. Again these panels show the cumulative RT distribution for all pre and post-lesion trials for 
each type of lesion. The overlapping nature of these distributions indicates no change between how quickly the birds responded before or after irrespective of their lesion.

\section{Discussion}

The present results demonstrate and further confirm the existence of a functional segregation of visual function between different regions of the entopallium. Lesions in the anterior portion of this structure significantly reduced localization accuracy in identifying color, and likely shape, targets in a texture discrimination. It also reduced the ability of the pigeons to discriminate among shapes requiring discrimination of their identity and relative location. However, it had no effect on performance of a different shape discrimination involving either dynamic or static presentation of the stimuli. In contrast, lesions in the posterior portion of this structure did significantly reduce the discrimination of these dynamic and static shapes. Also in contrast, these posterior lesions had little or no impact on the localization of color targets in the texture discrimination task. Like with the anterior lesions, the posterior lesions similarly affected their ability to localize shape targets in the localization task and similarly disrupted their accuracy in discrimination of the identity and location of different shapes. Finally, birds with the anterior lesions, but not the posterior lesions, responded faster in the localization tasks for both color and shape targets, while neither had an effect on RT in the where/what task. These different reactions across tasks with the different lesions indicate that the reductions in post-lesion performance are not due to any generalized effect of surgery or the temporal gap between pre and post-lesion testing, but are tied to the different attributes of the tasks. Hence, these contrasting functional profiles suggest that the different regions of this visual structure are processing different attributes of these complex visual signals.

Nguyen et al. (2004) suggested that the avian processing of visual information is segregated into separate visual streams that process different attributes. Our new findings are consistent with their results in that the anterior and posterior portions of the entopallium indeed seem to analyze different visual attributes. There are some interesting similarities in the results of the two studies. Similar to Nguyen et al. (2004) in which deficits were observed in a pattern detection task after anterior lesions, we found deficits in the texture localization task. In both tasks, the pigeons were required to detect and discriminate coarse shaped-based patterned regions. After the posterior lesions, Nguyen et al. (2004) found deficits in a coherent motion discrimination task, and we similarly found a selective deficit with this lesion in our one discrimination involving motion. In both cases, discriminations of moving shapes were involved. Thus, our results appear to suggest superficially that the anterior and posterior entopallium are involved in processing pattern and motion components of visual stimuli, respectively.

However, putative division among the regions cannot handle the entire pattern of our results First, the present results show that color discrimination is clearly affected by lesions in only the anterior entopallium (color perception was not examined in Nguyen et al. (2004)).

Deficits in color discrimination are in accordance with the anatomical fact that the anterior entopallium receives ascending thalamic projections from color-sensitive neurons in the nucleus rotundus (Wang et al., 1993; Laverghetta \& Shimizu, 2003). However, a previous 
study (Bessette \& Hodos, 1989) showed that only little or modest deficits were found on color discriminations tasks after lesions targeted the entire entopallium, presumably including the anterior portion. Since the extent of lesions in the anterior portion was not specifically analyzed, we speculate that incomplete damage in the anterior entopallium may be the reason of negligible effects on color perception in their study (Bessette \& Hodos, 1989). Second, in the present study posterior deficits were also observed in discriminations requiring the static discrimination of shapes and that did not involve motion. This shapebased deficit was also quite general in nature, as it was observed in all three tasks with this lesion. Because lesions in the posterior entopallium clearly affected the localization of shape targets, the discrimination of shape identity and location, and static discrimination of angular shape, this region must somehow be involved with shape processing as well as the processing of motion. Thus, it seems clear that a functional segregation within the entopallium exists, but these contrasts between these different studies, especially regarding the processing of shape and form, suggest complexities as to the identity of this division will require additional investigation.

Besides differences in accuracy, the present study showed for the first time that anterior, but not the posterior, lesions resulted in faster responses in localization tasks. Because no such changes were seen in other tasks, the differences in RT observed in the localization task was not a generalized outcome of the anterior lesion, but specifically tied to the combination of the task and lesion. These specific birds did not speed up all of their responding overall, but instead was damaged in a way that likely altered a process specifically tied to search performance. One possibility is that the birds were experiencing "illusory" targets in the displays that terminated the search process too soon. In this case, the perception of display may have been altered such that it produced "signals" consistent with what the birds had previously been responding to, such as misperceived edges. Another possibility is that the birds may have shifted their response criterion as to what was an acceptable target. If so, this criterion shift was limited to just the localization task, and not, for instance, the where/what task where speed of responding did not similar shift.

One complicating factor in these discriminations is that these various channels of visual information, such as color, shape and motion, are intimately tied together. For instance, it is not possible to have a moving stimulus that does not have a carrier form with color or brightness differences. Thus, the motion task deficits observed in Nguyen et al. (2004), for example, might have been due to a reduced shape-based capacity to see the small dots that carried the coherent motion that was not picked up by their pattern detection task. Likewise, our birds with the anterior lesions might have not been able to perceive the color of visual stimuli as well as before the lesion. Thus, any reduced color perception might have made it more difficult to see the shapes of the small elements. Such interactions among visual dimensions are important to keep in mind when interpreting results. One important attribute of our multiple task approach is that it helps to isolate these different attributes in useful and revealing ways, such as the reduced processing of shape across all tasks with posterior lesions.

In addition to the anterior-posterior axis, the entopallium may also be functionally segregated along the medial-lateral axis, as suggested by Hodos, Weiss, and Bessette (1986). 
In their study, the authors investigated the limits of the pigeons' ability to detect differences in stimulus size and then conducted selective lesion effects of either the medial or lateral entopllium. Based on the results, the authors suggested that the medial portion is involved in the processing of visual information with low spatial-frequency components whereas the lateral portion is involved in the high frequency components of information. Altogether, these studies indicate a more complex picture of functional segregation within the entopallium than the dichotomy suggested by Nguyen et al. (2004) or our results.

The present study does have has several limitations that should be noted. First, the number of animals was relatively small ( $\mathrm{n}=2$ for each lesion group) for such studies. Because of our use of three different tasks requiring months of pre-lesion training, it was to some extent prohibitive to include more subjects in testing our approach. Second, since the lesions were made electrolytically, both cell bodies and passing fibers were damaged. It is possible that lesions in the anterior or posterior portion of the entopallium inadvertently destroyed axons outside the target portion of the entopallium. Further studies are warranted to circumvent these issues by increasing the numbers of subjects and using chemical lesion methods.

Nonetheless, the multiple task approach used here and also by Nguyen et al. (2004) looks very promising as a tool for isolating how the different functions of the entopallium and their role in the processing and acting upon visual information. It is very likely that just as in mammals, birds also "divide and conquer" the processing of complex visual information by separating it into different streams within the brain. Constructing a better understanding how and why visual systems have evolved to separately process different visual attributes remains an important question to address.

\section{References}

Asen YL, Cook RG. Discrimination and categorization of actions by pigeons. Psychol. Sci. 2012; 23:617-624. [PubMed: 22539333]

Cook RG. Acquisition and transfer of visual texture discriminations by pigeons. J. Exp. Psychol. Anim. B. 1992a; 18:341-353.

Cook RG. Dimensional organization and texture discrimination in pigeons. J. Exp. Psychol. Anim. B. 1992b; 18:351-363.

Cook, RG. The visual perception and processing of textures by pigeons. In: Honig, WK.; Fetterman, JG., editors. Cognitive aspects of stimulus control. New Jersey: Lawrence Erlbaum Associates, Inc.; 1992c. p. 279-299.

Cook RG. The comparative psychology of avian visual cognition. Curr. Dir.In Psychol. Sci. 2000; 9:83-89.

Cook RG, Cavoto BR, Katz JS, Cavoto KK. Pigeon perception and discrimination of rapidly changing texture stimuli. J. Exp. Psychol. Anim. B. 1997; 23:390-400.

Cook RG, Cavoto KK, Cavoto BR. Mechanisms of multidimensional grouping, fusion, and search in avian texture discrimination. Anim. Learn Behav. 1996; 24:150-167.

Cook, RG.; Hagmann, CE. Grouping and early visual processing in avian vision. In: Lazareva, OF.; Shimizu, T.; Wasserman, EA., editors. How Animals See the World: Comparative Behavior, Biology, and Evolution of Vision. New York: Oxford University Press; 2012. p. 43-61.

Cook RG, Roberts S. The role of video coherence on object-based motion discriminations by pigeons. J. Exp. Psychol. Anim. B. 2007; 33:287-298.

Cook RG, Shaw R, Blaisdell AP. Dynamic object perception by pigeons: Discrimination of action in video presentations. Anim. Cogn. 2001; 4:137-146. [PubMed: 24777503] 
Hodos W. Color discrimination deficits after lesions of the nucleus rotundus in pigeons. Brain Behav. Evolut. 1969; 2:185-200.

Hodos, W. The visual capabilities of birds. In: Zeigler, HP.; Bischof, H-J., editors. Vision, brain, and behavior in birds. Massachusetts: The MIT Press; 1993. p. 77-98.

Hodos W, Karten HJ. Brightness and pattern discrimination deficits in the pigeon after lesions of the nucleus rotundus. Exp. Brain Res. 1966; 22:151-167. [PubMed: 5334899]

Hodos W, Weiss SRB, Bessette BB. Intensity difference thresholds after lesions of ectostriatum in pigeons. Behav. Brain Res. 1986; 21:203-214. [PubMed: 3768136]

Hodos W, Weiss SRB, Bessette BB. Intensity difference thresholds after lesions of ectostriatum in pigeons. Behav. Brain Res. 1988; 30:43-53. [PubMed: 3166707]

Karten HJ, Cox K, Mpodozis J. Two distinct populations of tectal neurons have unique connections within the retinotectorotundal pathway of the pigeon (Columba livia). J. Comp. Neurol. 1997; 387:449-465. [PubMed: 9335427]

Karten, HJ.; Hodos, W. A Stereotaxic Atlas of the Brain of the Pigeon (Columba livia). Maryland: Johns Hopkins Press; 1967. p. 193

Karten HJ, Hodos W. Telencephalic projections of the nucleus rotundus in the pigeon (Columba livia). J. Comp. Neurol. 1970; 140:35-51. [PubMed: 5459211]

Kellman PJ, Short KR. Development of three-dimensional form perception. J. Exp. Psychol. Human. $1987 ; 13: 545$.

Kirkpatrick-Steger K, Wasserman EA. The what and the where of the pigeon's processing of complex visual stimuli. J. Exp. Psychol. Anim. B. 1996; 22:60-67.

Koban AC, Cook RG. Rotational object discrimination by pigeons. J. Exp. Psychol. Anim. B. 2009; $35: 250-265$.

Kirkpatrick-Steger K, Wasserman EA. The what and where of the pigeon's processing of complex visual stimuli. J. Exp. Psychol. Anim. B. 1996; 22:60-67.

Krützfeldt NO, Wild JM. Definition and connections of the entopallium in the zebra finch (Taeniopygia guttata). J. Comp. Neurol. 2004; 468:452-465. [PubMed: 14681937]

Krützfeldt NO, Wild JM. Definition and novel connections of the entopallium in the pigeon (Columba livia). J. Comp. Neurol. 2004; 490:40-56. [PubMed: 16041718]

Laverghetta AV, Shimizu T. Organization of the ectostriatum based on afferent connections in the zebra finch (Taeniopygia guttata). Brain Res. 2003; 963:101-112. [PubMed: 12560115]

Lazareva, OF.; Shimizu, T.; Wasserman, Ea, editors. How Animals See the World: Comparative Behavior, Biology, and Evolution of Vision. New York: Oxford University Press; p. 543

Mpodozis J, Cox K, Shimizu T, Bischof HJ, Woodson W, Karten HJ. GABAergic inputs to the nucleus rotundus (pulvinar inferior) of the pigeon (Columba livia). J. Comp. Neurol. 1996; 374:204-222. [PubMed: 8906494]

Shimizu T, Bowers AN. Visual circuits of the avian telencephalon: Evolutionary implications. Behav. Brain Res. 1999; 98:183-191. [PubMed: 10683106]

Shimizu, T.; Watanabe, S. The avian visual system: Overview. In: Lazareva, OF.; Shimizu, T.; Wasserman, EA., editors. How Animals See the World: Comparative Behavior, Biology, and Evolution of Vision. New York: Oxford University Press; 2012. p. 473-482.

Ungerleider, LG.; Mishkin, M. Two cortical visual systems: Separation of appearance and location of objects. In: Ingle, DL.; Goodale, MA.; Mansfield, RJw, editors. Analysis of visual behavior. Massachusetts: The MIT Press; 1982. p. 549-586.

Wang YC, Jiang S, Frost BJ. Visual processing in pigeon nucleus rotundus: luminance, color, motion, and looming subdivisions. Visual Neurosci. 1993; 10:21-30.

Watanabe S, Bischof HJ. On the structure and function of the tectofugal visual pathway in laterally eyed birds. Eur. J. Morphol. 1997; 35:246-254. [PubMed: 9290933] 


\section{Texture-Based Target Localization}
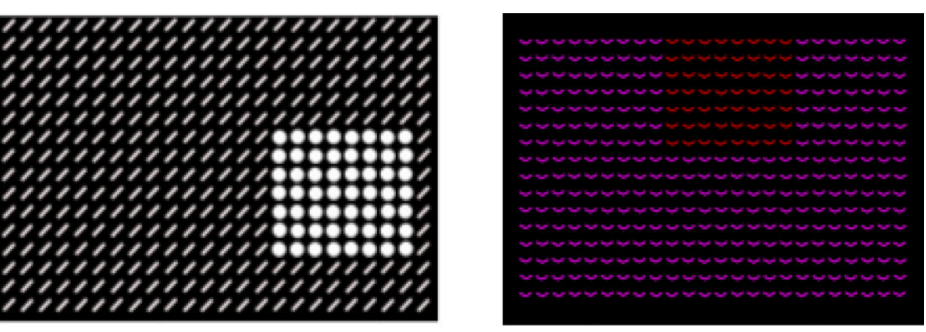

\section{Dynamic Shape Discrimination}
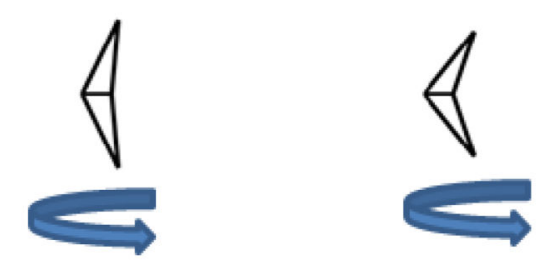

\section{Where/What Discrimination}

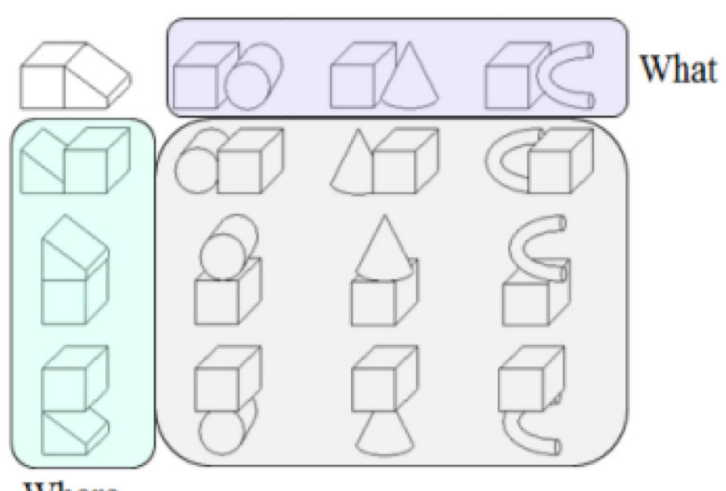

Where

Figure 1.

Representative examples of the stimuli tested in the three different tasks. The top row shows example of shape and color-based texture displays used in the localization task. The variably-located target could be made from different combinations of eight colors and eight shapes. The middle row shows examples of the two shapes tested in the dynamic shape discrimination task. On each trial, a single shape was randomly located in different random orientations on the monitor. The bottom row shows the set of 16 two-shape compounds used in the where/what task. For each bird, a different stimulus was designated correct. When paired with different foils, the task required the pigeon to discriminate the identity and relative locations of the shapes. 


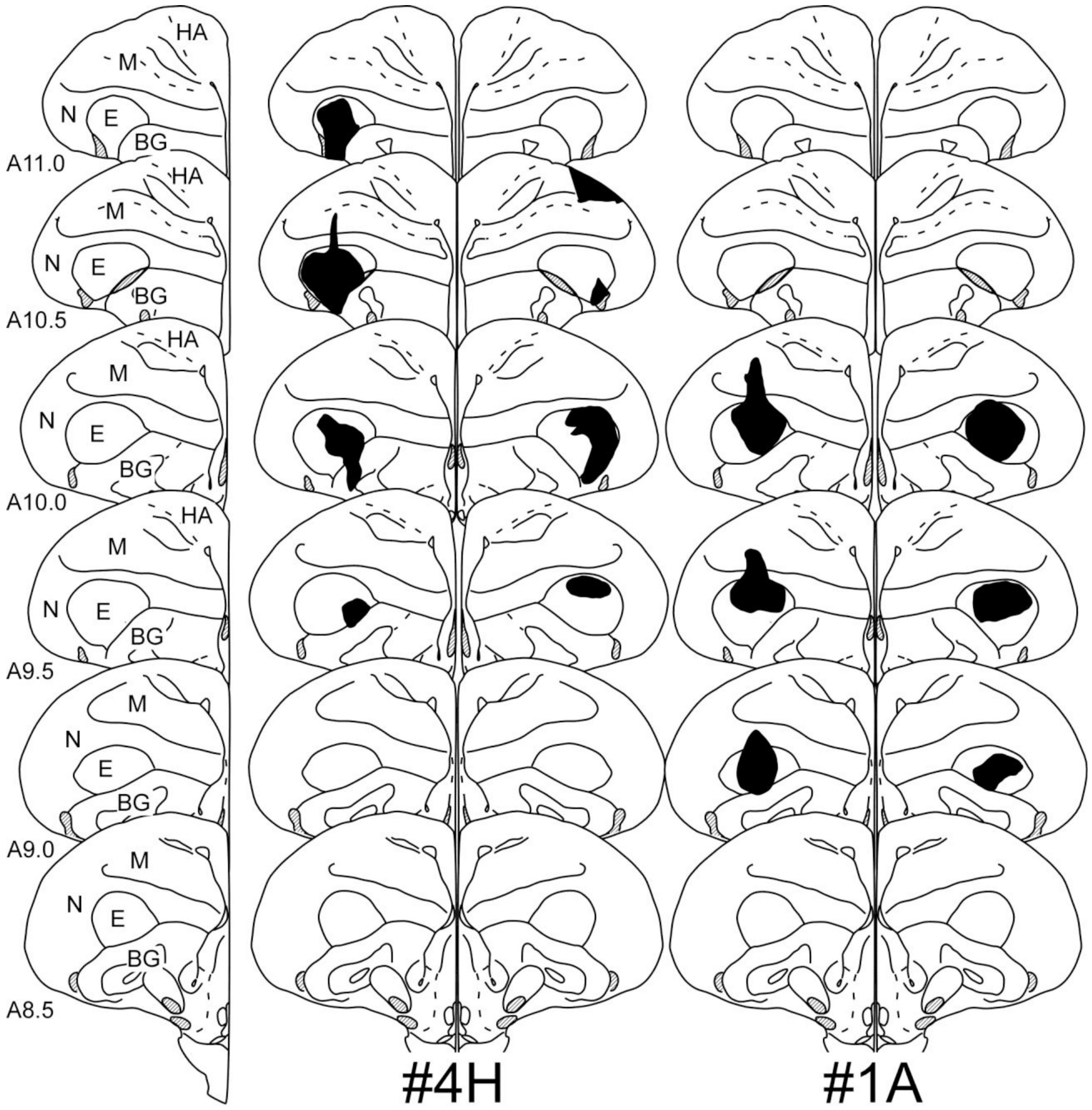

Figure 2.

Transverse sections through the telencephalon of \#4H in the anterior entopallial lesion group and \#1A in the posterior entopallial lesion group. The numbers A11.0 - A8.5 indicate the anterior-posterior coordinates. Black areas represent regions of necrosis/gliosis. BG: basal ganglia; E: entopallium; HA: hyperpallium apicale; M: mesopallium, N: nidopallium. 

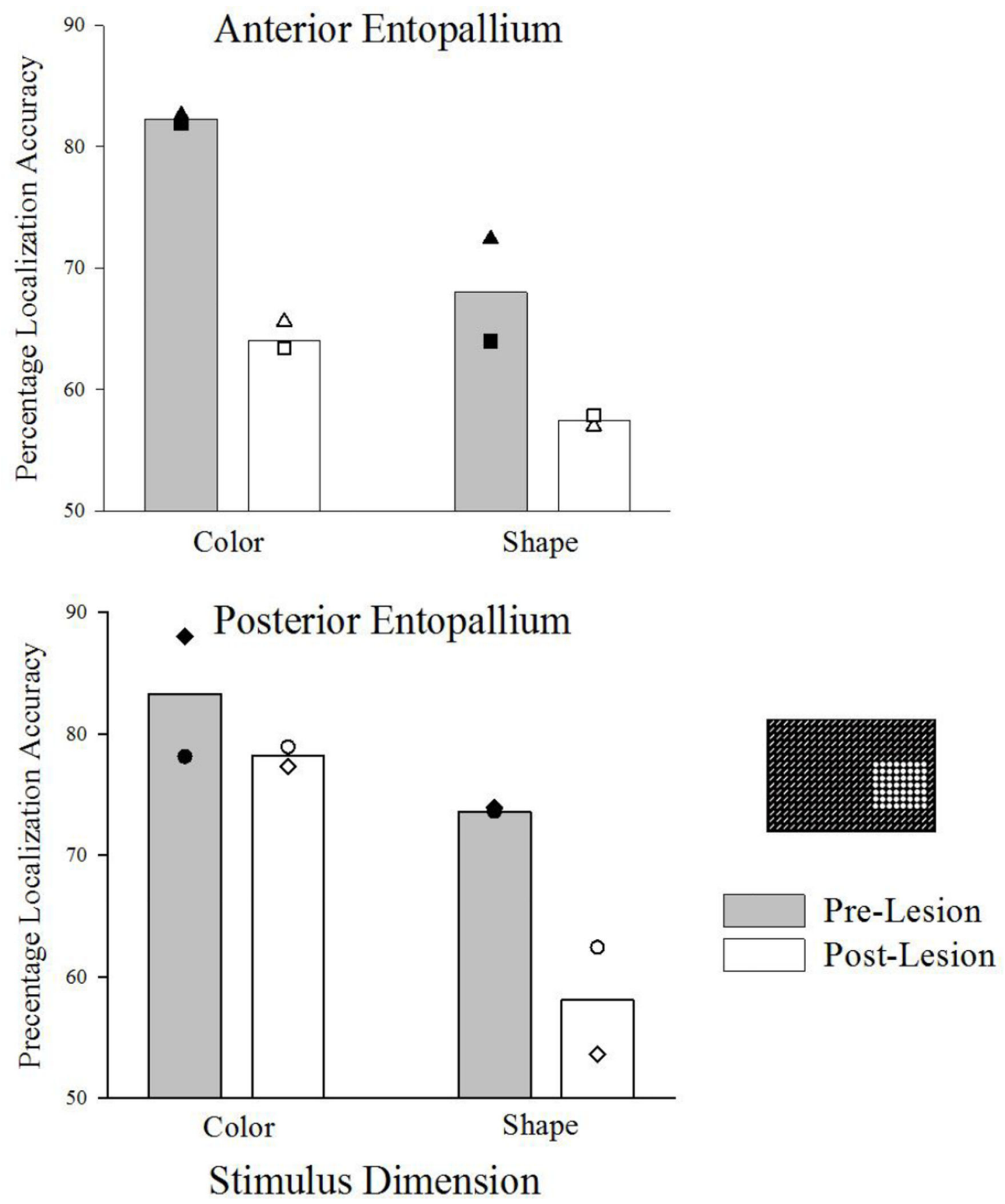

Figure 3.

Shown are the pre- and post-lesion color and shape target localization accuracies in the texture-based localization task for each type of lesion. The bars show average performance in each condition, while the individual symbols show the results for each pigeon individually. 
Anterior Entopallium
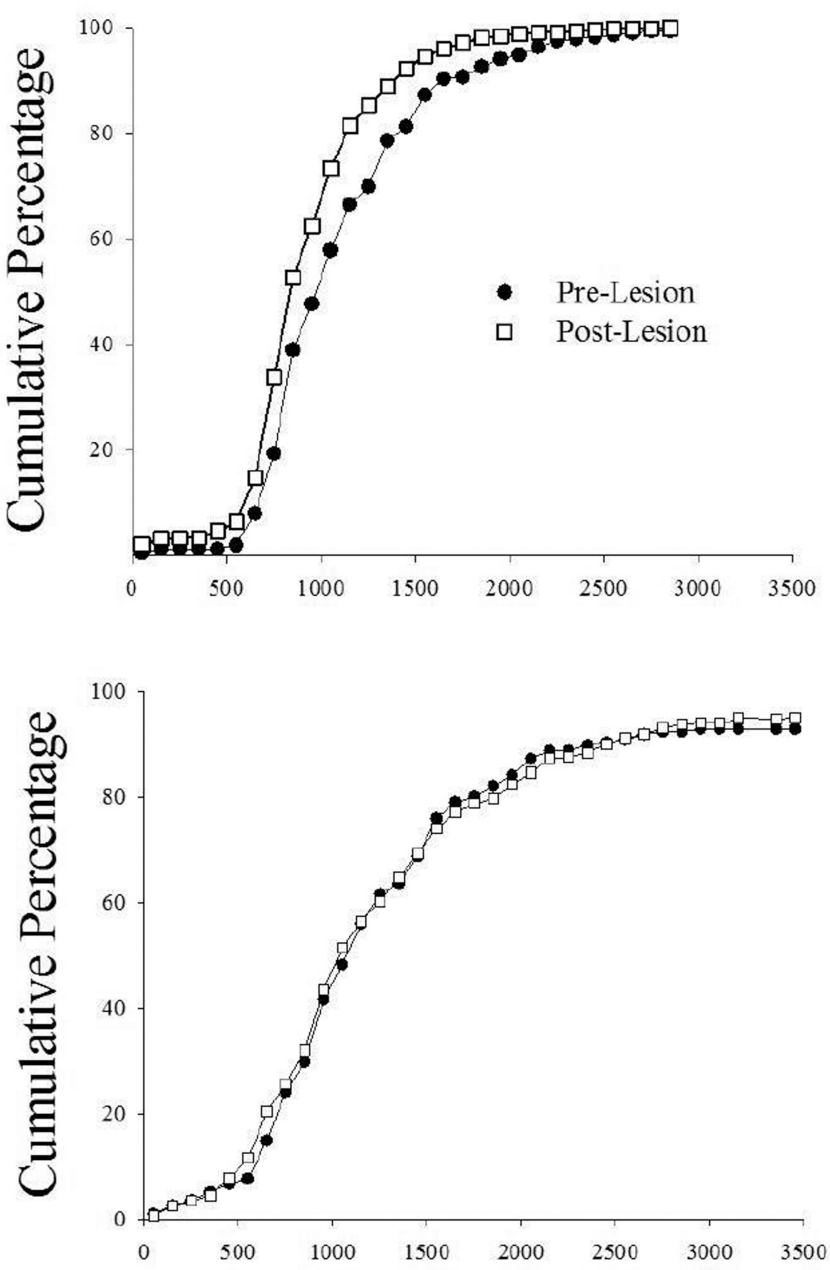

\section{Reaction Time}

Posterior Entopallium
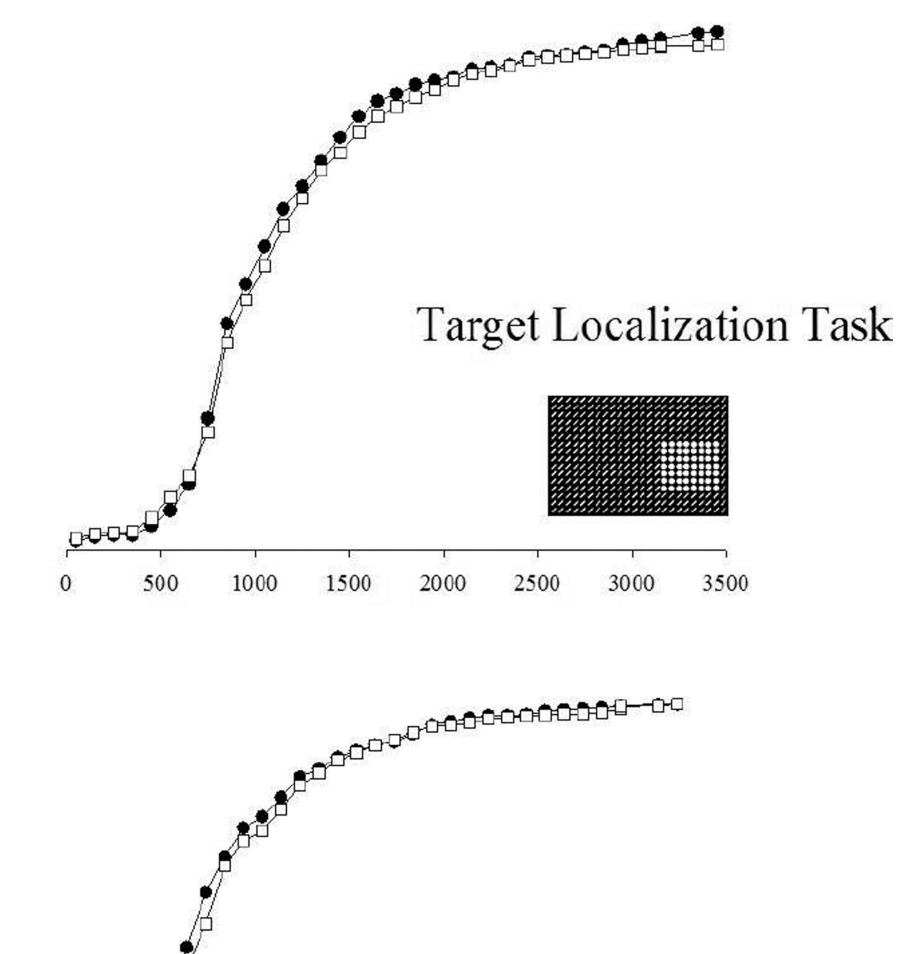

Where/What Task

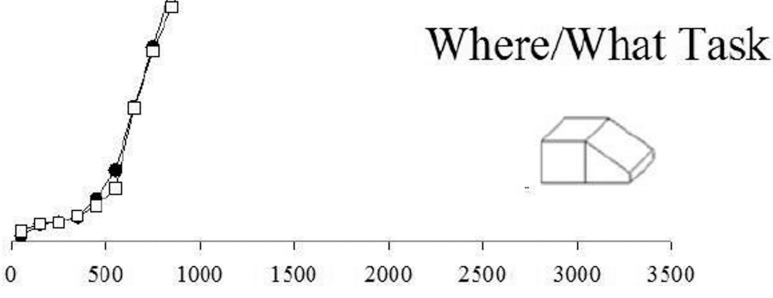

\section{Reaction Time}

Figure 4.

Shown are the pre- and post-lesion cumulative reaction time distributions in two different tasks. The top row shows the reaction times for the texture localization task, while the bottom row shows comparable data from the where/what task. 

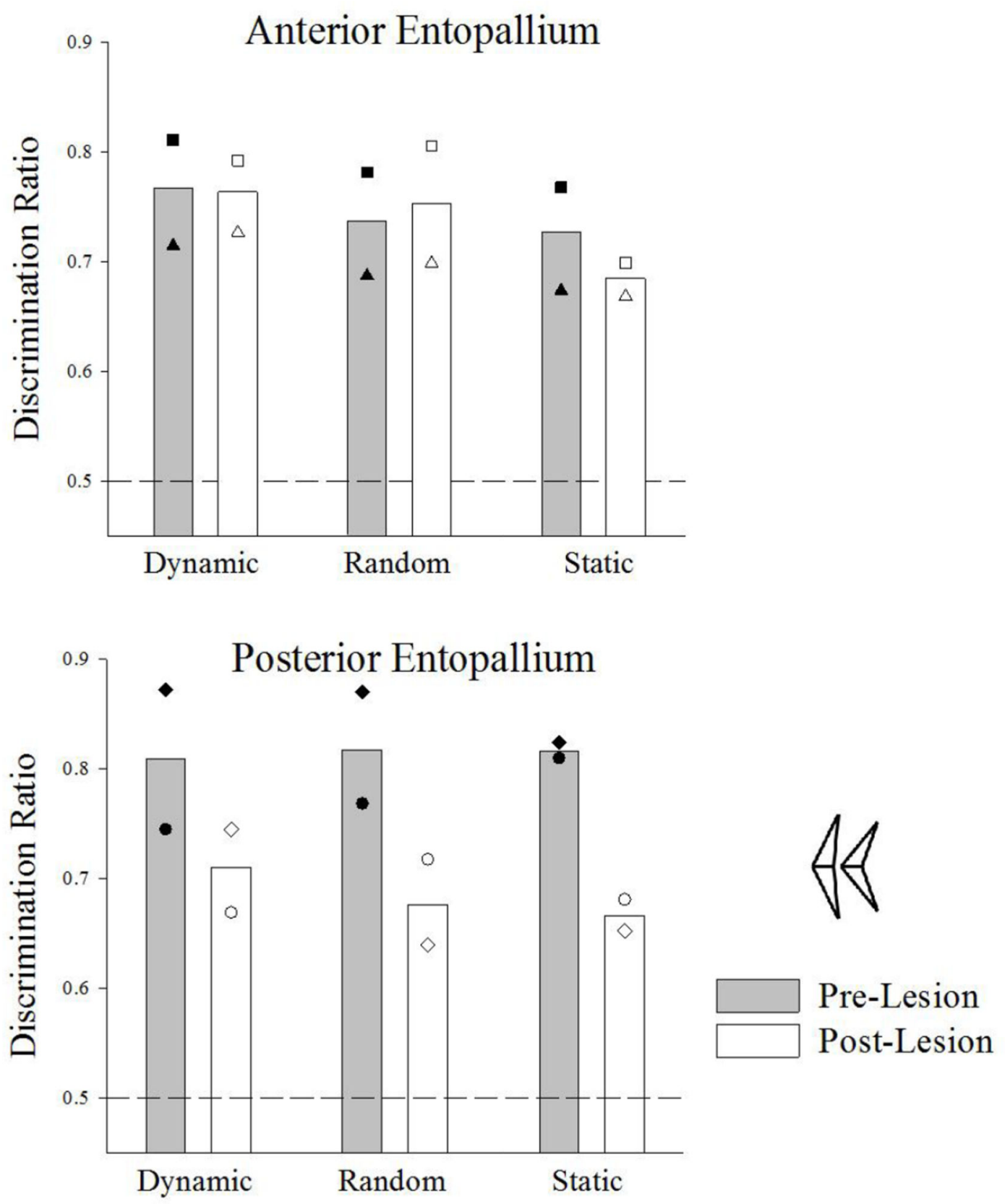

\section{Object Presentation Condition}

Figure 5.

Shown are the pre- and post-lesion discrimination ratios in the dynamic shape discrimination for each type of lesion. The bars show average performance in each condition, while the individual symbols show the results for each pigeon individually. The dashed line shows chance discrimination. 

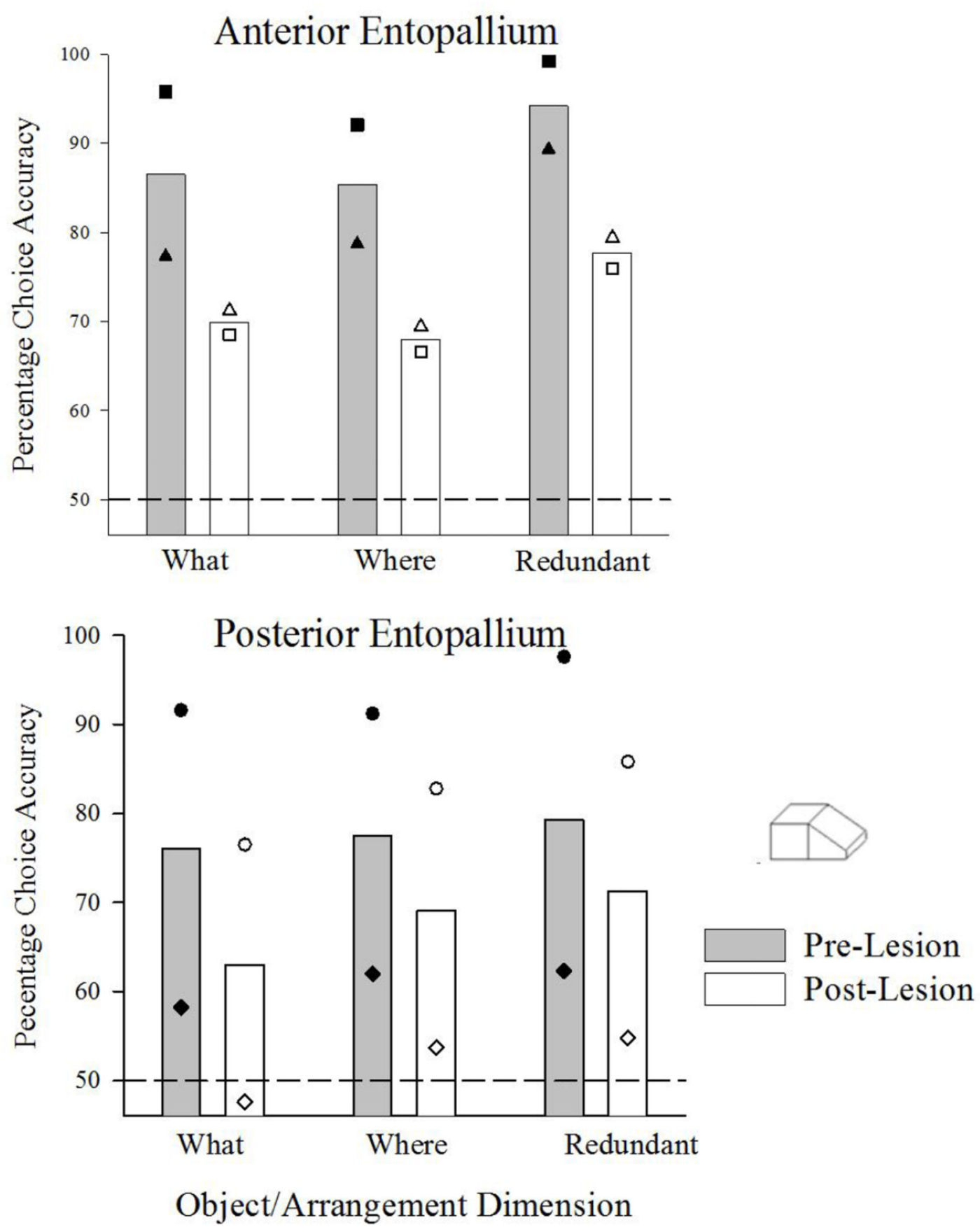

Figure 6.

Shown are the pre- and post-lesion choice accuracies in the where/what discrimination task for each type of lesion. The bars show average performance, while the individual symbols show the results for each pigeon individually. The dashed line shows chance discrimination. 